\title{
Atributos químicos de Latossolo após sucessivas aplicações de composto orgânico de dejeto líquido de suínos
}

\author{
Cledimar Rogério Lourenzi(1), Eloi Erhard Scherer(2), Carlos Alberto Ceretta ${ }^{(3)}$, Tadeu Luis Tiecher ${ }^{(3)}$, \\ Adriana Cancian ${ }^{(3)}$, Paulo Ademar Avelar Ferreira( ${ }^{(3)}$ e Gustavo Brunetto ${ }^{(3)}$
}

\begin{abstract}
(1)Universidade Federal de Santa Catarina, Centro de Ciências Agrárias, Departamento de Engenharia Rural, Rodovia Admar Gonzaga, no 1.346, Itacorubi, CEP 88034-000 Florianópolis, SC, Brasil. E-mail: lourenzicr@gmail.com (2)Empresa de Pesquisa Agropecuária e Extensão Rural de Santa Catarina, Centro de Pesquisa para Agricultura Familiar, Servidão Ferdinando Tusset, s/no, São Cristóvão, CEP 89801-970 Chapecó, SC, Brasil. E-mail: escherer@yahoo.com.br ${ }^{(3)}$ Universidade Federal de Santa Maria, Centro de Ciências Rurais, Departamento de Solos, Avenida Roraima, № 1.000, Cidade Universitária, Camobi, CEP 97105-900 Santa Maria, RS, Brasil. E-mail: carlosceretta@ufsm.br, tadeu.t@hotmail.com, cancian.adriana@gmail.com, ferreira.aap@gmail.com, brunetto.gustavo@gmail.com
\end{abstract}

Resumo - O objetivo deste trabalho foi determinar as alterações em atributos químicos de um Latossolo Vermelho após aplicações sucessivas de composto orgânico de dejeto líquido de suínos com maravalha, em sistema plantio direto. O experimento foi conduzido em Chapecó, SC, em Latossolo Vermelho, de setembro de 2005 a agosto de 2011. Foram aplicadas anualmente as doses de 0, 2, 4, 8 e $16 \mathrm{Mg} \mathrm{ha}^{-1}$ de composto de dejeto líquido de suínos com maravalha, além de um tratamento com adubação mineral (NPK). Após seis aplicações, coletaram-se amostras de solo nas camadas de 0-4, 4-8, 8-12, 12-16, 16-20, 20-40 e 40-60 cm e avaliaram-se os atributos químicos. As maiores doses do composto orgânico incrementaram o $\mathrm{pH}$ do solo e reduziram a acidez potencial. As aplicações de composto orgânico derivado de dejeto de suínos e maravalha promoveram alterações nos atributos químicos do Latossolo Vermelho avaliado, especialmente nas camadas superficiais do solo, com maior ênfase para aumento do $\mathrm{pH}$ do solo e dos teores de $\mathrm{P}, \mathrm{K}, \mathrm{Cu}$ e $\mathrm{Zn}$. A utilização de $4 \mathrm{Mg} \mathrm{ha}^{-1}$ do composto mantém os atributos químicos do solo semelhantes aos do tratamento NPK, que seguiu recomendação oficial para os estados do Rio Grande do Sul e de Santa Catarina, além de manter os teores de P nas camadas superficiais dentro do limite crítico ambiental para solos com altos teores de argila.

Termos para indexação: acidez do solo, acúmulo de nutrientes, fertilizante orgânico, resíduos orgânicos.

\section{Chemical attributes of an Oxisol after successive applications of organic compound with pig slurry}

\begin{abstract}
The objective of this work was to determine changes in the chemical attributes of an Oxisol after successive applications of organic compounds with pig slurry and wood shavings, under a no-tillage system. The experiment was conducted in the municipality of Chapecó, in the state of Santa Catarina, Brazil, in an Oxisol, from September 2005 to August 2011. The doses of $0,2,4,8$, and $16 \mathrm{Mg} \mathrm{ha}^{-1}$ compound with pig slurry and shavings were applied annually, as well as a treatment with mineral fertilizer (NPK). After six applications, soil was collected at the $0-4,4-8,8-12,12-16,16-20,20-40$, and 40-60-cm layers and the chemical attributes were evaluated. The highest doses of the organic compound increased soil $\mathrm{pH}$ and reduced potential acidity. The applications of the organic compound derived from swine manure and wood shavings promoted changes in the chemical attributes of the evaluated Oxisol, especially in the upper soil layers, with greater emphasis on increased soil $\mathrm{pH}$ and $\mathrm{P}, \mathrm{K}, \mathrm{Cu}$, and $\mathrm{Zn}$ contents. The use of $4 \mathrm{Mg} \mathrm{ha}^{-1}$ of the compound maintains the soil chemical attributes similar to those of the NPK treatment, which followed official recommendation for the states of Rio Grande do Sul and Santa Catarina, besides keeping the P content in the surface layers within the environmental critical limit for soils with high clay content.
\end{abstract}

Index terms: soil acidity, nutrient accumulation, organic fertilizer, organic waste.

\section{Introdução}

A suinocultura é amplamente difundida e desenvolvida no Brasil, especialmente nos estados da região Sul do País (Instituto Brasileiro de Geografia e Estatística, 2011). A atividade concentra-se em pequenas propriedades agrícolas, com predomínio de mão de obra familiar, e é o principal gerador de renda destes estabelecimentos (Seganfredo \& Girotto, 2004). Contudo, essa atividade apresenta elevado 
ônus ambiental, devido ao grande volume de dejetos gerados.

No sistema convencional de criação, os dejetos de suínos normalmente são armazenados em esterqueiras anaeróbicas e aplicados na superfície do solo para o suprimento de nutrientes às plantas. Entretanto, o baixo teor de matéria seca normalmente dificulta e encarece sua distribuição no campo. Uma alternativa que vem sendo utilizada é a produção de composto orgânico a partir da combinação desses dejetos com maravalha, pois resíduos sólidos normalmente apresentam maiores concentrações de matéria seca e nutrientes do que o dejeto líquido de suínos (Giacomini \& Aita, 2008; Scherer, 2013), o que facilita seu transporte e sua distribuição.

A aplicação do dejeto na forma sólida, como composto, ou líquida em áreas em sistema plantio direto ocorre na superfície do solo, sem incorporação (Scherer, 2013), e a distribuição é realizada frequentemente em áreas restritas e próximas dos locais de armazenamento. Essa prática, ao longo dos anos, pode promover alterações em atributos químicos do solo relacionados à acidez, como, por exemplo, aumento dos valores de $\mathrm{pH}$ do solo e diminuição dos teores de $\mathrm{Al}$ ou da saturação por esse nutriente (Ceretta et al., 2003; Lourenzi et al., 2011; Brunetto et al., 2012).

A aplicação de resíduos orgânicos também pode promover, principalmente nas camadas mais superficiais do solo, o incremento dos teores de nutrientes como $\mathrm{P}, \mathrm{K}, \mathrm{Ca}$ e $\mathrm{Mg}$ (McDowell et al., 2001; Gatiboni et al., 2008; Ceretta et al., 2010a; Guardini et al., 2012; Lourenzi et al., 2013). Com isso, há melhoria no ambiente químico do solo para o crescimento de raízes, por causa do aumento da saturação por bases e da redução da saturação por Al (Lourenzi et al., 2011; Brunetto et al., 2012). No entanto, também pode ocorrer o aumento dos teores de metais pesados no solo, como $\mathrm{Cu}$ e $\mathrm{Zn}$ (Girotto et al., 2010; Mattias et al., 2010; Basso et al., 2012; Popovic \& Jensen, 2012; Tiecher et al., 2013), remanescentes de rações consumidas pelos suínos. Isso porque em torno de $90 \%$ do $\mathrm{Cu}$ e do $\mathrm{Zn}$ ingeridos pelos suínos por meio dos suplementos minerais são excretados nas fezes e na urina, estando presentes nos dejetos (Aldrich et al., 2002; Nicholson et al., 2003). Dessa forma, aplicações sucessivas de resíduos orgânicos, muitas vezes com suprimento de nutrientes acima das necessidades das culturas, podem promover acúmulos excessivos de nutrientes, como $\mathrm{N}, \mathrm{P}, \mathrm{Cu}$ e $\mathrm{Zn}$, no solo, o que potencializa a transferência desses elementos por escoamento superficial e percolação, e, consequentemente, contamina mananciais de águas superficiais e subsuperficiais (Bergström \& Kirchmann, 2006; Ceretta et al., 2010b; Girotto et al., 2013).

A aplicação de resíduos orgânicos também representa adição de carbono ao solo que pode causar mudanças quantitativas e qualitativas na matéria orgânica do solo (Hernández et al., 2006; Lourenzi et al., 2011). Isso, somado ao incremento do teor de nutrientes no solo, proporciona maiores produções de matéria seca pelas culturas (Lourenzi et al., 2014).

A maioria dos trabalhos encontrados na literatura abordam os efeitos do uso de dejetos de suínos in natura, sem nenhum tipo de processamento, sobre os atributos químicos do solo (Adeli et al., 2008; Scherer et al., 2010; Brunetto et al., 2012). Portanto, é importante avaliar os efeitos do uso de composto orgânico produzido com dejetos de suínos sobre os atributos químicos do solo, uma vez que as características desses resíduos são diferentes das dos dejetos líquidos, sobretudo quanto à carga orgânica e à forma como os nutrientes são aplicados ao solo.

O objetivo deste trabalho foi determinar as alterações em atributos químicos de um Latossolo Vermelho após aplicações sucessivas de composto orgânico de dejeto líquido de suínos com maravalha, em sistema plantio direto.

\section{Material e Métodos}

O trabalho foi conduzido durante seis anos, de setembro de 2005 a agosto de 2011, no município de Chapecó, SC $\left(27^{\circ} 05^{\prime} 47^{\prime \prime S}, 52^{\circ} 37^{\prime} 06^{\prime \prime} \mathrm{W}\right.$, a $674 \mathrm{~m}$ de altitude), em Latossolo Vermelho distroférrico típico (Santos et al., 2013). Na época de instalação do experimento, a área apresentava histórico de pelo menos dois anos em sistema plantio direto. Nesse momento, o solo foi amostrado na camada de $0-10 \mathrm{~cm} \mathrm{e}$ apresentava os seguintes atributos: $834 \mathrm{~g} \mathrm{~kg}^{-1}$ de argila; $112 \mathrm{~g} \mathrm{~kg}^{-1}$ de silte; $54 \mathrm{~g} \mathrm{~kg}^{-1}$ de areia; $42 \mathrm{~g} \mathrm{~kg}^{-1}$ de matéria orgânica; $\mathrm{pH}$ em $\mathrm{H}_{2} \mathrm{O}$ de 6,2; índice SMP de 6,2; 8,5 e $220 \mathrm{mg} \mathrm{kg}^{-1}$ de $\mathrm{P}$ e $\mathrm{K}$ disponíveis, respectivamente, extraídos por Mehlich-1; 8,2 e 4,6 cmol $_{\mathrm{c}} \mathrm{dm}^{-3}$ de Ca e $\mathrm{Mg}$ trocáveis, respectivamente, extraídos por $\mathrm{KCl}$ 
$1,0 \mathrm{~mol} \mathrm{~L}^{-1} ; 3,5 \mathrm{cmol}_{\mathrm{c}} \mathrm{dm}^{-3}$ de $\mathrm{H}+\mathrm{Al} ; \mathrm{CTC}_{\mathrm{pH} 7,0}$ de $17 \mathrm{cmol}_{\mathrm{c}} \mathrm{dm}^{-3}$; e saturação por bases e por $\mathrm{Al}$ de $79 \mathrm{e}$ $0 \%$, respectivamente. O clima da região é do tipo Cfa, subtropical úmido, de acordo com a classificação de Köppen. As médias anuais de temperatura, precipitação e umidade relativa do ar foram: $18,8^{\circ} \mathrm{C}, 1.954 \mathrm{~mm}$ e $72 \%$, respectivamente.

Os tratamentos consistiram das doses de $0,2,4,8 \mathrm{e}$ $16 \mathrm{Mg} \mathrm{ha}^{-1}$ de composto orgânico derivado de dejeto líquido de suínos com maravalha, além de adubação mineral (NPK), como recomendado pelo Manual... (2004). O composto foi produzido em plataforma de compostagem seguindo a metodologia de Scherer et al. (2009), com a adição de, aproximadamente, $10 \mathrm{~L}$ de dejeto líquido de suínos para cada quilograma de maravalha. As características dos compostos aplicados em cada ano agrícola foram obtidas por meio de análises realizadas de acordo com Tedesco et al. (1995) e estão apresentadas na Tabela 1. Já as quantidades de nutrientes adicionadas em cada tratamento estão apresentadas na Tabela 2. Em todos os anos, foi implantada uma sucessão com as culturas de milho (Zea mays L.) no verão, feijão-preto (Phaseolus vulgaris L.) na safrinha e aveia-preta (Avena strigosa L.) no inverno; os tratamentos foram aplicados sempre antes da semeadura do milho, distribuídos sobre a superfície do solo sem incorporação e na área total da parcela. Utilizou-se o delineamento experimental de blocos ao acaso com quatro repetições, e cada parcela apresentava dimensões de $5 \times 5 \mathrm{~m}$, o que totalizou $25 \mathrm{~m}^{2}$. Em agosto de 2011, 11 meses após a última aplicação do composto, foram coletadas amostras de solo através da abertura de uma trincheira até $60 \mathrm{~cm}$ de profundidade, com auxílio de uma pá de corte. As camadas amostradas foram: $0-4,4-8,8-12,12-$ $16,16-20,20-40$ e $40-60 \mathrm{~cm}$. O solo foi seco ao ar, moído, passado em peneira com malha de $2 \mathrm{~mm}$ e armazenado.

Nas amostras de solo, foram analisados: valores de pH em $\mathrm{H}_{2} \mathrm{O}$ (relação 1:1); índice SMP; teores de P e K disponíveis, extraídos por Mehlich-1; teores de Al, Ca e Mg trocáveis, extraídos por $\mathrm{KCl}$ 1,0 $\mathrm{mol} \mathrm{L}^{-1}$ (Tedesco et al., 1995); teores de Cu eZn disponíveis, extraídos por EDTA 0,01 mol L-1 (Chaignon et al., 2003); e carbono orgânico (Claessen, 1997), cujo teor foi multiplicado

Tabela 1. Características dos compostos utilizados em cada ano agrícola de condução do experimento ${ }^{(1)}$.

\begin{tabular}{|c|c|c|c|c|c|c|c|c|c|c|}
\hline \multirow[t]{2}{*}{ Ano } & MS & $\mathrm{N}$ & $\mathrm{P}_{2} \mathrm{O}_{5}$ & $\mathrm{~K}_{2} \mathrm{O}$ & $\mathrm{Ca}$ & $\mathrm{Mg}$ & $\mathrm{Cu}$ & $\mathrm{Zn}$ & $\mathrm{Mn}$ & $\mathrm{pH}$ \\
\hline & \multicolumn{6}{|c|}{ - } & \multicolumn{4}{|c|}{ 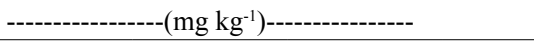 } \\
\hline 2005 & 30 & 1,37 & 1,67 & 1,12 & 0,38 & 0,32 & 123 & 218 & 368 & 6,8 \\
\hline 2006 & 45 & 1,48 & 2,04 & 1,18 & 0,82 & 0,38 & 272 & 364 & 585 & 8,2 \\
\hline 2007 & 50 & 1,38 & 2,18 & 1,34 & 0,86 & 0,62 & 302 & 385 & 512 & 7,6 \\
\hline 2008 & 41 & 1,84 & 3,08 & 1,42 & 2,80 & 0,72 & 203 & 572 & 621 & 7,2 \\
\hline 2009 & 37 & 1,08 & 1,89 & 1,18 & 1,44 & 0,27 & 127 & 126 & 149 & 6,8 \\
\hline 2010 & 38 & 1,19 & 1,66 & 0,78 & 0,90 & 0,27 & 230 & 270 & 301 & 6,3 \\
\hline
\end{tabular}

(1)As concentrações dos nutrientes no composto foram calculadas em base seca. MS, matéria seca.

Tabela 2. Quantidades totais de nutrientes aplicados durante os seis anos de condução do experimento, com aplicações anuais do composto de dejeto líquido de suínos com maravalha e adubação mineral.

\begin{tabular}{|c|c|c|c|c|c|c|c|}
\hline $\begin{array}{l}\text { Composto }^{(1)} \\
\left(\mathrm{Mg} \mathrm{ha}^{-1}\right)\end{array}$ & N & $\mathrm{P}_{2} \mathrm{O}_{5}$ & $\mathrm{~K}_{2} \mathrm{O}$ & $\begin{array}{c}\mathrm{Ca} \\
\left.\mathrm{kg} \mathrm{ha}^{-1}\right)\end{array}$ & $\mathrm{Mg}$ & $\mathrm{Cu}$ & $\mathrm{Zn}$ \\
\hline 0 & - & - & - & - & - & - & - \\
\hline 2 & 167 & 250 & 140 & 144 & 52 & 2,51 & 3,87 \\
\hline 4 & 334 & 501 & 281 & 288 & 103 & 5,03 & 7,74 \\
\hline 8 & 668 & 1.002 & 562 & 576 & 206 & 10,06 & 15,48 \\
\hline 16 & 1.336 & 2.003 & 1.123 & 1.152 & 412 & 20,11 & 30,96 \\
\hline $\mathrm{NPK}^{(2)}$ & 780 & 480 & 480 & - & - & - & - \\
\hline
\end{tabular}

${ }^{(1)}$ Composto orgânico de dejeto líquido de suínos com maravalha. ${ }^{(2)}$ Adubação mineral recomendada pelo Manual... (2004). 
por 1,724 (fator de Van Bemmelen), para obter os teores de matéria orgânica do solo (MOS). Após as extrações, o teor de P disponível foi determinado de acordo com a metodologia proposta por Murphy \& Riley (1962). $\mathrm{O} \mathrm{K}$ disponível foi determinado em fotômetro de chama, modelo B262 (Micronal, S.A., São Paulo, SP), enquanto o $\mathrm{Al}$ trocável foi determinado por meio de titulação com $\mathrm{NaOH} 0,0025 \mathrm{~mol} \mathrm{~L}^{-1}$ e o $\mathrm{Ca}$, o $\mathrm{Mg}$, o $\mathrm{Cu}$ e o Zn, em espectrofotômetro de absorção atômica. A partir dos dados obtidos, calcularam-se a capacidade de troca de cátions a $\mathrm{pH} 7,0\left(\mathrm{CTC}_{\mathrm{pH} 7,0}\right)$, a saturação por alumínio, a saturação por bases e a acidez potencial ( $\mathrm{H}+\mathrm{Al})$, com uso das equações descritas no Manual... (2004).

Os dados de cada variável, dentro da mesma camada nos diferentes tratamentos, foram submetidos à análise de variância (Anova), tendo-se utilizado o teste $\mathrm{F}$, a $5 \%$ de probabilidade. Quando observada diferença significativa entre os tratamentos, na mesma camada, realizou-se análise de regressão, tendo-se considerado apenas os tratamentos que receberam as doses do composto $\left(0,2,4,8\right.$ e $\left.16 \mathrm{Mg} \mathrm{ha}^{-1}\right)$. Todas as análises foram realizadas com uso do programa Sisvar (Universidade Federal de Lavras, Lavras, MG).

\section{Resultados e Discussão}

As aplicações do composto promoveram aumentos no valor de $\mathrm{pH}$ do solo até a camada de $12-16 \mathrm{~cm}$ de profundidade, principalmente quando a dose de $16 \mathrm{Mg} \mathrm{ha}^{-1}$ foi utilizada (Tabela 3 e Figura $1 \mathrm{~A}$ ). Esses resultados podem ser atribuídos à adsorção de íons $\mathrm{H}^{+}$ nos resíduos culturais em decomposição, que fazem parte da fração não húmica da MOS, e, também, aos grupos funcionais da MOS, especialmente das frações ácidos húmicos e ácidos fúlvicos (Lourenzi et al., 2011). Além disso, as propriedades alcalinas e a dissociação de carbonatos presentes nos dejetos também podem contribuir para a elevação dos valores de $\mathrm{pH}$ em solos que recebem aplicações de dejetos (Chantigny et al., 2004).

A utilização de dejetos de suínos, tanto na forma de cama sobreposta quanto na líquida, é capaz de elevar o $\mathrm{pH}$ em $\mathrm{H}_{2} \mathrm{O}$ do solo, como observado por Brunetto et al. (2012), em trabalho desenvolvido em Argissolo Vermelho, no município de Braço do Norte, SC. Esses autores avaliaram, durante oito anos, o efeito de doses de cama sobreposta e de dejeto líquido de suínos para suprir 90 e $180 \mathrm{~kg} \mathrm{ha}^{-1}$ de $\mathrm{N}$ para as culturas estudadas, tendo constatado maiores valores de $\mathrm{pH}$ do solo nos tratamentos com aplicação de cama sobreposta de suínos. Esse resultado pode ser explicado pela maior presença de compostos orgânicos nesses resíduos, o que favorece a adsorção de íons $\mathrm{H}^{+}$e, consequentemente, promove aumento do $\mathrm{pH}$ do solo. Nessa mesma linha, Lourenzi et al. (2011), após 19 aplicações das doses de $0,20,40$ e $80 \mathrm{~m}^{3} \mathrm{ha}^{-1}$ de dejeto líquido de suínos em Argissolo Vermelho, relataram aumento no valor do $\mathrm{pH}$ do solo até $8 \mathrm{~cm}$ de profundidade, com uso da maior dose de dejeto.

Para os teores de $\mathrm{H}+\mathrm{Al}$, não foram observadas diferenças entre os tratamentos até a profundidade de $8 \mathrm{~cm}$; entretanto, abaixo desta camada, os tratamentos que receberam aplicações do composto e de NPK apresentaram menores valores do que a área que não recebeu as aplicações (Tabela 3 e Figura 1 B). As calagens realizadas em anos anteriores aos da condução do experimento, sem incorporação, parecem ter sido mais decisivas para os baixos valores de $\mathrm{H}+\mathrm{Al}$ até $8,0 \mathrm{~cm}$ de profundidade. Isso porque, abaixo desta camada, tanto a aplicação do composto quanto de NPK proporcionaram diminuição na acidez potencial (Figura 1 B). Resultados semelhantes foram obtidos por Brunetto et al. (2012), que, ao utilizar doses de cama sobreposta e de dejeto líquido de suínos equivalentes a 90 e $180 \mathrm{~kg} \mathrm{ha}^{-1}$ de $\mathrm{N}$ em Argissolo Vermelho, não verificaram efeito das aplicações dos tratamentos sobre os teores de $\mathrm{H}+\mathrm{Al}$ até $10 \mathrm{~cm}$ de profundidade.

A utilização do composto durante os seis anos experimentais proporcionou incrementos de 22, 26 e $23 \%$ nos teores de MOS, com a aplicação de 4, 8 e $16 \mathrm{Mg} \mathrm{ha}^{-1}$, respectivamente, na camada de $0-4 \mathrm{~cm}$ (Tabela 3 e Figura $1 \mathrm{C}$ ). Esses resultados podem ser atribuídos à adição de grandes quantidades de matéria seca via composto, o que aumenta o aporte de resíduos orgânicos sobre a superfície do solo. A deposição do composto orgânico sobre os restos culturais das plantas diminui a área de contato com o solo, o que retarda a atividade da biomassa microbiana e, por consequência, a mineralização do composto orgânico (Falleiro et al., 2003). Além disso, as grandes quantidades de nutrientes aplicadas nas maiores doses de composto também podem favorecer maiores produções de matéria seca pelas culturas, por exemplo, de milho, aveia-preta e feijão, como relatado por Lourenzi et al. (2014). Assim, o composto aumenta o aporte de resíduos vegetais na 
superfície do solo e, consequentemente, incrementa os teores de matéria orgânica (Adeli et al., 2008; Lourenzi et al., 2011).

$\mathrm{O}$ aumento dos teores de matéria orgânica se refletiu em incrementos da $\mathrm{CTC}_{\mathrm{pH} 7,0}$, além de manter a saturação por bases alta e a saturação por Al baixa até a camada de 8-12 cm, como pôde ser observado nos tratamentos com a aplicação de 4,8 e $16 \mathrm{Mg} \mathrm{ha}^{-1}$ do composto (Tabela 3 e Figuras 1 D, E e F). Esses resultados são semelhantes aos encontrados por Brunetto et al. (2012), que testaram a aplicação de doses de cama sobreposta e de dejeto líquido de suínos em Argissolo Vermelho, e constataram estreita relação entre os teores de matéria orgânica do solo e a $\mathrm{CTC}_{\mathrm{pH} 7,0}$ do solo. Esses autores salientam que o aumento da $\mathrm{CTC}_{\mathrm{pH} 7,0}$ do solo favorece a adsorção de cátions presentes nos dejetos, como K, $\mathrm{Ca}$ e $\mathrm{Mg}$.

As aplicações do composto e de NPK proporcionaram incrementos nos teores de $\mathrm{P}$ disponível no solo até a camada de 8-12 cm. No entanto, os incrementos foram mais relevantes com uso de $16 \mathrm{Mg} \mathrm{ha}^{-1}$ do composto, quando avaliada a camada de $0-4 \mathrm{~cm}$, na qual os aumentos foram de 2.120 e $350 \%$ em relação ao solo com $0 \mathrm{Mg} \mathrm{ha}^{-1}$ do composto e com a aplicação de NPK, respectivamente (Tabela 3 e Figura $2 \mathrm{~A}$ ). Cabe destacar

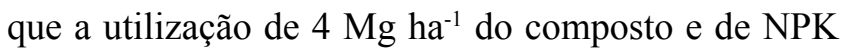

Tabela 3. Análise de regressão ${ }^{(1)}$ em camadas de Latossolo Vermelho após seis anos de utilização de composto orgânico de dejeto líquido de suínos com maravalha e adubação mineral (NPK).

\begin{tabular}{|c|c|c|c|c|c|c|}
\hline Camada $(\mathrm{cm})$ & Equação & $\mathrm{R}^{2}$ & Equação & $\mathrm{R}^{2}$ & Equação & $\mathrm{R}^{2}$ \\
\hline & \multicolumn{2}{|l|}{$\mathrm{pH} \mathrm{em} \mathrm{H}_{2} \mathrm{O}$} & \multicolumn{2}{|l|}{$\mathrm{H}+\mathrm{Al}$} & \multicolumn{2}{|c|}{ Matéria orgânica } \\
\hline $0-4$ & $\hat{y}=6,724+0,017 x$ & $0,83 * *$ & ns & & $\hat{y}=56,541+3,339 x-0,1526 x^{2}$ & $0,88^{* *}$ \\
\hline $4-8$ & $\hat{y}=6,120+0,065 x-0,0025 x^{2}$ & $0,91 *$ & ns & & ns & \\
\hline $8-12$ & $\hat{y}=5,622+0,081 x-0,0038 x^{2}$ & $0,82 * *$ & $\hat{y}=7,313-0,601 x+0,0269 x^{2}$ & $0,86^{* *}$ & ns & \\
\hline $12-16$ & $\hat{y}=5,342+0,017 x$ & $0,29 * *$ & $\hat{y}=9,850-0,2306 x$ & $0,44 * *$ & ns & \\
\hline $16-20$ & ns & & $\hat{y}=16,211-1,094 x+0,0451 x^{2}$ & $0,71 * *$ & ns & \\
\hline $20-40$ & ns & & $\hat{y}=24,999-1,671 x+0,0707 x^{2}$ & $0,79 * *$ & ns & \\
\hline \multirow[t]{2}{*}{$40-60$} & $\hat{y}=4,437+0,056 x-0,0021 x^{2}$ & $0,82 *$ & $\hat{y}=29,096-1,946 x+0,0875 x^{2}$ & $0,96 * *$ & ns & \\
\hline & \multicolumn{2}{|c|}{$\mathrm{CTC}_{\mathrm{pH} 7,0}$} & \multicolumn{2}{|c|}{ Saturação por bases } & Saturação por Al & \\
\hline $0-4$ & $\hat{y}=14,408+1,183 x-0,0360 x^{2}$ & $0,93 * *$ & $\hat{y}=85,355+0,434 x$ & $0,81 * *$ & ns & \\
\hline $4-8$ & $\hat{y}=14,346+0,908 x-0,0371 x^{2}$ & $0,90 * *$ & $\hat{y}=71,903+2,821 x-0,1217 x^{2}$ & $0,98 * *$ & ns & \\
\hline $8-12$ & ns & & $\hat{y}=54,722+4,235 x-0,1941 x^{2}$ & $0,97 * *$ & ns & \\
\hline $12-16$ & $\hat{y}=17,331+0,338 x-0,0281 x^{2}$ & $0,53^{*}$ & $\hat{y}=43,837+3,292 x-0,1398 x^{2}$ & $0,53 * *$ & ns & \\
\hline $16-20$ & $\hat{y}=21,700-0,339 x$ & $0,83 * *$ & $\hat{y}=27,589+3,061 x-0,1459 x^{2}$ & $0,46 * *$ & ns & \\
\hline $20-40$ & $\hat{y}=27,844-1,182 x+0,0413 x^{2}$ & $0,87 * *$ & $\hat{y}=10,723+2,642 x-0,1423 x^{2}$ & $0,46^{* *}$ & $\hat{y}=63,478-2,558 x+0,1794 x^{2}$ & $0,60 * *$ \\
\hline \multirow[t]{2}{*}{$40-60$} & $\hat{y}=31,191-1,735 x+0,0747 x^{2}$ & $0,96 * *$ & $\hat{y}=6,650+1,418 x-0,0758 x^{2}$ & $0,98 * *$ & $\hat{y}=72,618-0,910 x+0,0868 x^{2}$ & $0,79 *$ \\
\hline & \multicolumn{2}{|c|}{ Fósforo } & \multicolumn{2}{|c|}{ Potássio } & \multicolumn{2}{|c|}{ Cobre } \\
\hline $0-4$ & $\hat{y}=3,789+10,761 x+0,1295 x^{2}$ & $0,99 *$ & $\hat{y}=216,875+61,641 x-2,0818 x^{2}$ & ${ }^{2} 0,96 * *$ & $\hat{y}=5,338+1,121 x-0,0148 x^{2}$ & $0,99 *$ \\
\hline $4-8$ & $\hat{y}=1,122+2,583 x$ & $0,98 * *$ & $\hat{y}=134,227+46,251 x-1,5328 x^{2}$ & ${ }^{2} 0,96 * *$ & $\hat{y}=4,863+0,130 x$ & $0,85^{* *}$ \\
\hline $8-12$ & $\hat{y}=2,775+0,863 x$ & $0,89 * *$ & $\hat{y}=96,191+16,946 x$ & $0,94 * *$ & $\hat{y}=4,267+0,078 x$ & $0,63^{* *}$ \\
\hline $12-16$ & ns & & $\hat{y}=52,612+14,031 x$ & $0,98 * *$ & ns & \\
\hline $16-20$ & ns & & $\hat{y}=42,384+8,558 x$ & $0,95 * *$ & ns & \\
\hline $20-40$ & ns & & ns & & ns & \\
\hline \multirow[t]{2}{*}{$40-60$} & ns & & ns & & ns & \\
\hline & \multicolumn{2}{|l|}{ Zinco } & \multicolumn{2}{|l|}{ Cálcio } & \multicolumn{2}{|l|}{ Magnésio } \\
\hline $0-4$ & $\hat{y}=6,510+2,942 x-0,0462 x^{2}$ & $0,99 * *$ & $\hat{y}=6,666+0,765 x-0,0305 x^{2}$ & $0,89 * *$ & $\hat{y}=4,911+0,272 x$ & $0,95 * *$ \\
\hline $4-8$ & $\hat{y}=4,349+0,179 x$ & $0,77 *$ & $\hat{y}=5,427+0,696 x-0,0334 x^{2}$ & $0,94 * *$ & $\hat{y}=4,456+0,376 x-0,0118 x^{2}$ & $0,93^{* *}$ \\
\hline $8-12$ & ns & & $\hat{y}=4,388+0,485 x-0,0253 x^{2}$ & $0,89 * *$ & $\hat{y}=4,058+0,400 x-0,0178 x^{2}$ & $0,83^{* *}$ \\
\hline $12-16$ & ns & & $\hat{y}=3,284+0,380 x-0,0216 x^{2}$ & $0,50 * *$ & $\hat{y}=3,650+0,373 x-0,0199 x^{2}$ & $0,67 * *$ \\
\hline $16-20$ & ns & & $\hat{y}=2,599+0,207 x-0,0134 x^{2}$ & $0,32 * *$ & $\hat{y}=3,314+0,242 x-0,0144 x^{2}$ & $0,36^{* *}$ \\
\hline $20-40$ & ns & & $\hat{y}=1,200+0,253 x-0,0165 x^{2}$ & $0,59 * *$ & $\hat{y}=1,541+0,242 x-0,0134 x^{2}$ & $0,41^{* *}$ \\
\hline $40-60$ & ns & & ns & & ns & \\
\hline
\end{tabular}

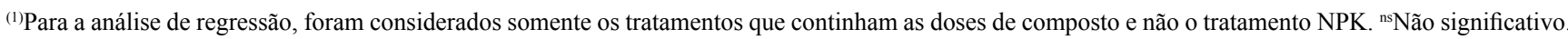
* e**Significativo a 5 e $1 \%$ de probabilidade, respectivamente. 

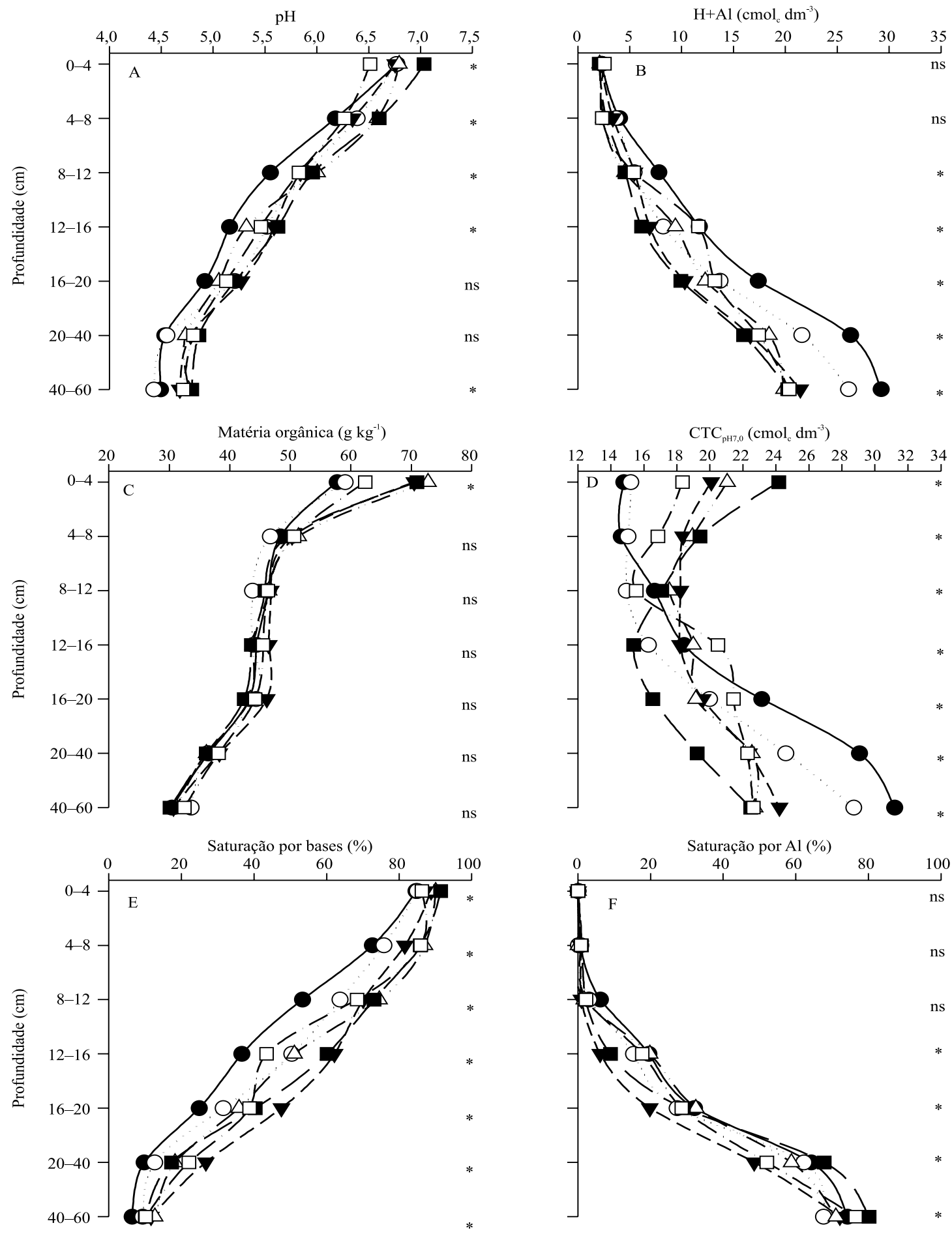

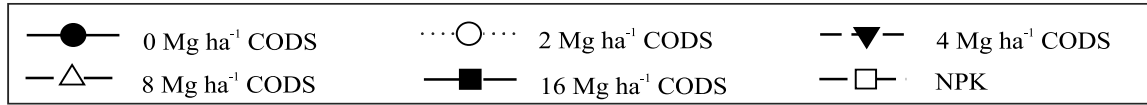

Figura 1. Valores de $\mathrm{pH}$ em $\mathrm{H}_{2} \mathrm{O}(\mathrm{A}) ; \mathrm{H}+\mathrm{Al}(\mathrm{B})$; matéria orgânica do solo (C); $\mathrm{CTC}_{\mathrm{pH} 7,0}(\mathrm{D})$; saturação por bases (E) e saturação por alumínio (F) em camadas de Latossolo Vermelho após seis anos de utilização de composto orgânico de dejeto líquido de suínos com maravalha (CODS) e adubação mineral (NPK). ${ }^{\text {ns }}$ Não significativo. *Diferença significativa pelo teste F, a $5 \%$ de probabilidade. 

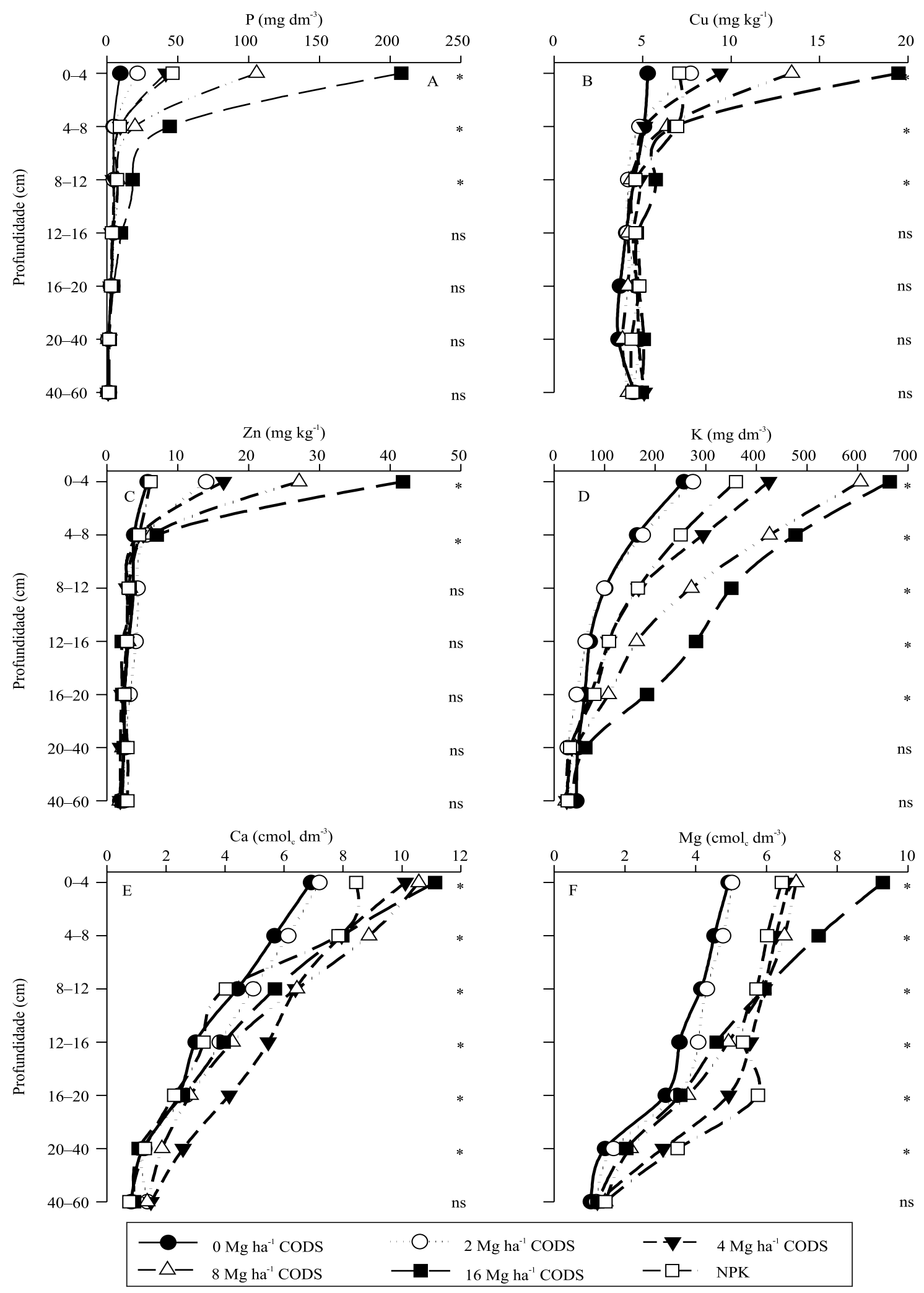

Figura 2. Teores de fósforo (A); cobre (B); zinco (C); potássio (D); cálcio (E) e magnésio (F) em camadas de Latossolo Vermelho após seis anos de utilização de composto orgânico de dejeto líquido de suínos com maravalha (CODS) e adubação mineral (NPK). ns Não significativo. *Diferença significativa pelo teste F, a $5 \%$ de probabilidade. 
promoveu incrementos semelhantes de $\mathrm{P}$ disponível nas camadas de solo avaliadas, tendo adicionado ao solo quantidades de $\mathrm{P}_{2} \mathrm{O}_{5}$ similares, de 501 e $480 \mathrm{~kg} \mathrm{ha}^{-1}$, respectivamente.

Os maiores teores de $\mathrm{P}$ disponível observados nas camadas superficiais do solo podem ser atribuídos à aplicação superficial e sem incorporação do composto $\mathrm{e}$, principalmente, às quantidades de $\mathrm{P}$ aplicadas junto com as doses de 8 e $16 \mathrm{Mg} \mathrm{ha}^{-1}$ do composto, que foram 108 e $317 \%$ maiores do que a recomendada pelo Manual... (2004), que é de $480 \mathrm{~kg} \mathrm{ha}^{-1} \mathrm{de}$ P. Nesse sentido, os resultados obtidos no presente trabalho estão muito próximos aos do trabalho de Guardini et al. (2012), em Argissolo Vermelho. Embora os teores de $\mathrm{P}$ na camada de $0-10 \mathrm{~cm}$ estejam abaixo de $118 \mathrm{mg} \mathrm{dm}^{-3}$, considerado como limite crítico ambiental em solos com teor de argila $>80 \%$, segundo Gatiboni et al. (2014), quando analisada apenas a camada de $0-4 \mathrm{~cm}$ da dose de $16 \mathrm{Mg} \mathrm{ha}^{-1}$ do composto, os teores de $\mathrm{P}$ estão acima do limite crítico ambiental, o que evidencia o risco de contaminação dos recursos hídricos.

Os teores de $\mathrm{Cu}$ e $\mathrm{Zn}$ foram incrementados com uso do composto até as camadas de $8-12 \mathrm{~cm}$ e de 4-8 cm, respectivamente, e os maiores teores foram observados com a aplicação de $16 \mathrm{Mg} \mathrm{ha}^{-1}$ do composto (Tabela 3 e Figuras 2 B e C). Em termos relativos, os teores de $\mathrm{Cu}$ e $\mathrm{Zn}$ na camada de $0-4 \mathrm{~cm}$ do solo, com uso de $16 \mathrm{Mg} \mathrm{ha}^{-1}$ do composto, foram 268 e $629 \%$ maiores, respectivamente, que os obtidos no solo com $0 \mathrm{Mg} \mathrm{ha}^{-1}$ do composto. Chama a atenção o fato de ter havido incremento de $\mathrm{Cu}$ até a camada de $4-8 \mathrm{~cm}$, quando utilizou-se NPK, em comparação ao solo com $0 \mathrm{Mg} \mathrm{ha}^{-1}$ do composto (Figura $2 \mathrm{~B}$ ).

Os maiores teores de $\mathrm{Cu}$ e $\mathrm{Zn}$ disponíveis nas camadas superficiais do solo com a aplicação do composto podem ser atribuídos à aplicação superficial e sem incorporação do composto, bem como ao aumento do teor de MOS até a camada de $0-4 \mathrm{~cm}$. Isso porque o $\mathrm{Cu}$ e o $\mathrm{Zn}$ apresentam afinidade por grupos funcionais da matéria orgânica, especialmente o $\mathrm{Cu}$ (Tiecher et al., 2013). Somado a isso, a maior quantidade de $\mathrm{Zn}$ aplicada com os compostos (Tabela 2) também justifica o incremento de $629 \%$ de $\mathrm{Zn}$ contra o de $268 \%$ de $\mathrm{Cu}$, na camada de $0-4 \mathrm{~cm}$, quando $16 \mathrm{Mg} \mathrm{ha}^{-1}$ do composto foram utilizados. $\mathrm{O}$ aumento dos teores de $\mathrm{Cu}$ e $\mathrm{Zn}$ nas camadas superficiais do solo torna esses elementos mais suscetíveis às transferências por escoamento superficial (Girotto et al., 2010) e, também, pode significar possibilidade de toxidez por $\mathrm{Cu}$ às plantas. Nesse sentido, Girotto et al. (2013) observaram toxidez por $\mathrm{Cu}$ em estudo que avaliou o cultivo de milho por 25 dias após a emergência das plantas, em Argissolo que recebeu 19 aplicações de dejeto líquido de suínos.

A aplicação das doses do composto proporcionou incrementos dos teores de $\mathrm{K}$ disponível até a camada de 16-20 cm (Tabela 3 e Figura 2 D). Na camada de 0-4 cm do solo, com a aplicação das doses de 8 e $16 \mathrm{Mg} \mathrm{ha}^{-1}$ do composto, os incrementos foram de 136 e $159 \%$, respectivamente, em relação à mesma camada do solo com $0 \mathrm{Mg} \mathrm{ha}^{-1}$ do composto. Porém, quando os teores de $\mathrm{K}$ disponível, desta mesma camada e nesses mesmos tratamentos, foram comparados ao solo que recebeu a aplicação de NPK, os incrementos foram de 69 e 85\%, respectivamente. Os teores trocáveis de $\mathrm{Ca}$ e $\mathrm{Mg}$ também aumentaram com a aplicação das doses do composto e de NPK, com incrementos até a camada de 20-40 cm (Tabela 3 e Figura 2 E e F). Com a aplicação da dose de $16 \mathrm{Mg} \mathrm{ha}^{-1}$ do composto, os teores trocáveis de $\mathrm{Ca}$ e $\mathrm{Mg}$ foram, respectivamente, 61 e $88 \%$ superiores aos do tratamento com $0 \mathrm{Mg} \mathrm{ha}^{-1}$ do composto, na camada de $0-4 \mathrm{~cm}$.

Os incrementos dos teores de $\mathrm{K}, \mathrm{Ca}$ e $\mathrm{Mg}$ observados, principalmente nas camadas superficiais do solo, podem ser atribuídos às quantidades desses elementos adicionadas ao solo com as aplicações do composto nos seis anos experimentais, que foram de $936,1.152$ e $413 \mathrm{~kg} \mathrm{ha}^{-1}$, respectivamente, com a dose de $16 \mathrm{Mg} \mathrm{ha}^{-1}$ do composto. Adeli et al. (2008) aplicaram dejetos de suínos ao solo durante 15 anos e observaram incrementos de K na solução do solo com o aumento de $\mathrm{Ca}$ e $\mathrm{Mg}$, bem como sua competição pelos sítios de adsorção na CTC do solo.

\section{Conclusões}

1. As aplicações de composto orgânico derivado de dejeto líquido de suínos e maravalha promovem alterações nos atributos químicos do Latossolo Vermelho avaliado, especialmente nas camadas superficiais do solo, com maior ênfase para aumento do $\mathrm{pH}$ do solo e dos teores de $\mathrm{P}, \mathrm{K}, \mathrm{Cu}$ e $\mathrm{Zn}$.

2. A utilização de $4 \mathrm{Mg}^{-1} \mathrm{a}^{-1}$ do composto mantém os atributos químicos do solo semelhantes aos do tratamento NPK, recomendado oficialmente para os estados do Rio Grande do Sul e de Santa Catarina, e, portanto, é a dose recomendada para condições semelhantes às do presente trabalho. 


\section{Agradecimentos}

À Coordenação de Aperfeiçoamento de Pessoal de Nível Superior (Capes) e ao Conselho Nacional de Desenvolvimento Científico e Tecnológico (CNPq), pela concessão de bolsas.

\section{Referências}

ADELI, A.; BOLSTER, C.H.; ROWE, D.E.; MCLAUGHLIN, M.R.; BRINK, G.E. Effect of long-term swine effluent application on selected soil properties. Soil Science, v.173, p.223-235, 2008. DOI: 10.1097/ss.0b013e31816408ae.

ALDRICH, A.P.; KISTLER, D.; SIGG, L. Speciation of $\mathrm{Cu}$ and $\mathrm{Zn}$ in drainage water from agricultural soils. Environmental Science and Technology, v.36, p.4824-4830, 2002. DOI: 10.1021/ es025813x.

BASSO, C.J.; CERETTA, C.A.; FLORES, E.M. de M.; GIROTTO, E. Teores totais de metais pesados no solo após aplicação de dejeto líquido de suínos. Ciência Rural, v.42, p.653-659, 2012. DOI: 10.1590/S0103-84782012000400012.

BERGSTRÖM, L.; KIRCHMANN, H. Leaching and crop uptake of nitrogen and phosphorus from pig slurry as affected by different application rates. Journal of Environmental Quality, v.35, p.1803-1811, 2006. DOI: 10.2134/jeq2006.0003.

BRUNETTO, G.; COMIN, J.J.; SCHMITT, D.E.; GUARDINI, R.; MEZZARI, C.P.; OLIVEIRA, B.S.; MORAES, M.P. de; GATIBONI, L.C.; LOVATO, P.E.; CERETTA, C.A. Changes in soil acidity and organic carbon in a sandy Typic Hapludalf after medium-term pig slurry and deep-litter application. Revista Brasileira de Ciência do Solo, v.36, p.1620-1628, 2012. DOI: 10.1590/S0100-06832012000500026.

CERETTA, C.A.; DURIGON, R.; BASSO, C.J.; BARCELLOS, L.A.R.; VIEIRA, F.C.B. Características químicas de solo sob aplicação de esterco líquido de suínos em pastagem natural. Pesquisa Agropecuária Brasileira, v.38, p.729-735, 2003. DOI: $10.1590 / \mathrm{S} 0100-204 X 2003000600009$.

CERETTA, C.A.; GIROTTO, E.; LOURENZI, C.R.; TRENTIN, G.; VIEIRA, R.C.B.; BRUNETTO, G. Nutrient transfer by runoff under no tillage in a soil treated with successive applications of pig slurry. Agriculture, Ecosystems and Environment, v.139, p.689-699, 2010b. DOI: 10.1016/j.agee.2010.10.016.

CERETTA, C.A.; LORENSINI, F.; BRUNETTO, G.; GIROTTO, E.; GATIBONI, L.C.; LOURENZI, C.R.; TIECHER, T.L.; DE CONTI, L.; TRENTIN, G.; MIOTTO, A. Frações de fósforo no solo após sucessivas aplicações de dejetos de suínos em plantio direto. Pesquisa Agropecuária Brasileira, v.45, p.593-602, 2010a. DOI: 10.1590/S0100-204X2010000600009.

CHAIGNON, V.; SANCHEZ-NEIRA, I.; HERRMANN, P.; JAILLARD, B.; HINSINGER, P. Copper bioavailability and extractability as related to chemical properties of contaminated soils from a vine-growing area. Environmental Pollution, v.123, p.229-238, 2003. DOI: 10.1016/S0269-7491(02)00374-3.
CHANTIGNY, M.H.; ROCHETTE, P.; ANGERS, D.A.; MASSEÉ, D.; CÔTE, D. Ammonia volatilization and selected soil characteristics following application of anaerobically digested pig slurry. Soil Science Society of America Journal, v.68, p.306-312, 2004. DOI: $10.2136 /$ sssaj2004.3060.

CLAESSEN, M.E.C. (Org.). Manual de métodos de análise de solo. 2.ed. rev. e atual. Rio de Janeiro: EMBRAPA-CNPS, 1997. 212p. (EMBRAPA-CNPS. Documentos, 1).

FALLEIRO, R.M.; SOUZA, C.M.; SILVA, C.S.W.; SEDIYAMA, C.S.; SILVA, A.A.; FAGUNDES, J.L. Influência dos sistemas de preparo nas propriedades químicas e físicas do solo. Revista Brasileira de Ciência do Solo, v.27, p.1097-1104, 2003. DOI: 10.1590/S0100-06832003000600014.

GATIBONI, L.C.; BRUNETTO, G.; KAMINSKI, J.; RHEINHEIMER, D. dos S.; CERETTA, C.A.; BASSO, C.J. Formas de fósforo no solo após sucessivas adições de dejeto líquido de suínos em pastagem natural. Revista Brasileira de Ciência do Solo, v.32, p.1753-1761, 2008. DOI: 10.1590/ S0100-06832008000400040.

GATIBONI, L.C.; SMYTH, T.J.; SCHMITT, D.E.; CASSOL, P.C.; OLIVEIRA, C.M.B. Proposta de limites críticos ambientais de fósforo para solos de Santa Catarina. Lages: UDESC-CAV, 2014. 38p. (CAV-UDESC. Boletim técnico, 2).

GIACOMINI, S.J.; AITA, C. Emissão de dióxido de carbono após aplicação de dejetos líquidos e cama sobreposta de suínos. Pesquisa Agropecuária Brasileira, v.43, p.107-114, 2008. DOI: 10.1590/S0100-204X2008000100014.

GIROTTO, E.; CERETTA, C.A.; BRUNETTO, G.; SANTOS, D.R. dos; SILVA, L.S. da; LOURENZI, C.R.; LORENSINI, F.; VIEIRA, R.C.B.; SCHMATZ, R. Acúmulo e formas de cobre e zinco no solo após aplicações sucessivas de dejeto líquido de suínos. Revista Brasileira de Ciência do Solo, v.34, p.955-965, 2010. DOI: $10.1590 / \mathrm{S} 0100-06832010000300037$.

GIROTTO, E.; CERETTA, C.A.; LOURENZI, C.R.; LORENSINI, F.; TIECHER, T.L.; VIEIRA, R.C.B.; TRENTIN, G.; BASSO, C.J.; MIOTTO, A.; BRUNETTO, G. Nutrient transfers by leaching in a no-tillage system through soil treated with repeated pig slurry applications. Nutrient Cycling Agroecosystems, v.95, p.115-131, 2013. DOI: $10.1007 / \mathrm{s} 10705-013-9552-2$.

GUARDINI, R.; COMIN, J.J.; SCHMITT, D.E.; TIECHER, T.; BENDER, M.A.; SANTOS, D.R. dos.; MEZZARI, C.P.; OLIVEIRA, B.S.; GATIBONI, L.C.; BRUNETTO, G. Accumulation of phosphorus fractions in typic Hapludalf soil after long-term application of pig slurry and deep pig litter in a no-tillage system. Nutrient Cycling Agroecosystems, v.93, p.215-225, 2012. DOI: $10.1007 / \mathrm{s} 10705-012-9511-3$.

HERNÁNDEZ, D.; PLAZA, C.; SENESI, N.; POLO, A. Detection of copper(II) and zinc(II) binding to humic acids from pig slurry and amended soils by fluorescence spectroscopy. Environmental Pollution, v.143, p.212-220, 2006. DOI: $10.1016 / \mathrm{j}$. envpol.2005.11.038.

INSTITUTO BRASILEIRO DE GEOGRAFIA E ESTATÍSTICA. Produção da pecuária municipal 2011. Rio de Janeiro, 2011. v. 39. Disponível em: <ftp://ftp.ibge.gov.br/Producao_Pecuaria/ 
Producao_da_Pecuaria_Municipal/2011/ppm2011.pdf>. Acesso em: 18 fev. 2014.

LOURENZI, C.R.; CERETTA, C.A.; BRUNETTO, G.; GIROTTO, E.; TIECHER, T.L.; VIEIRA, R.C.B.; CANCIAN, A.; FERREIRA, P.A.A. Pig slurry and nutrient accumulation and dry matter and grain yield in various crops. Revista Brasileira de Ciência do Solo, v.38, p.949-958, 2014. DOI: 10.1590/S0100-06832014000300027.

LOURENZI, C.R.; CERETTA, C.A.; SILVA, L.S. da; GIROTTO, E.; LORENSINI, F.; TIECHER, T.L.; DE CONTI, L.; TRENTIN, G.; BRUNETTO, G. Nutrients in soil layers under no-tillage after successive pig slurry applications. Revista Brasileira de Ciência do Solo, v.37, p.157-167, 2013. DOI: 10.1590/ S0100-06832013000100016.

LOURENZI, C.R.; CERETTA, C.A.; SILVA, L.S. da; TRENTIN, G.; GIROTTO, E.; LORENSINI, F.; TIECHER, T.L.; BRUNETTO, G. Soil chemical properties related to acidity under successive pig slurry applications. Revista Brasileira de Ciência do Solo, v.35, p.1827-1836, 2011. DOI: 10.1590/ S0100-06832011000500037.

MANUAL de adubação e calagem para os estados do Rio Grande do Sul e Santa Catarina. 10.ed. Porto Alegre: Sociedade Brasileira de Ciência do Solo, 2004. 394p.

MATTIAS, J.L.; CERETTA, C.A.; NESI, C.N.; GIROTTO, E.; TRENTIN, E.E.; LOURENZI, C.R.; VIEIRA, R.C.B. Copper, zinc and manganese in soils of two watersheds in Santa Catarina with intensive use of pig slurry. Revista Brasileira de Ciência do Solo, v.34, p.1445-1454, 2010. DOI: 10.1590/ S0100-06832010000400040.

MCDOWELL, R.; SHARPLEY, A.; FOLMAR, G. Phosphorus export from an agricultural watershed: linking source and transport mechanisms. Journal of Environmental Quality, v.30, p.1587-1595, 2001. DOI: 10.2134/jeq2001.3051587x.

MURPHY, J.; RILEY, J.P. A modified single solution method for the determination of phosphate in natural waters. Analytica Chimica Acta, v.27, p.31-36, 1962. DOI: 10.1016/S0003-2670(00)88444-5.

NICHOLSON, F.A.; SMITH, S.R.; ALLOWAY, B.J.; CARLTON-SMITH, C.; CHAMBERS, B.J. An inventory of heavy metals inputs to agricultural soils in England and Wales. Science of the Total Environment, v.311, p.205-219, 2003. DOI: 10.1016/ S0048-9697(03)00139-6.

POPOVIC, O.; JENSEN, L.S. Storage temperature affects distribution of carbon, VFA, ammonia, phosphorus, copper and zinc in raw pig slurry and its separated liquid fraction. Water Research, v.46, p.3849-3858, 2012. DOI: 10.1016/j.watres.2012.04.020.

SANTOS, H.G. dos; JACOMINE, P.K.T.; ANJOS, L.H.C. dos; OLIVEIRA, V.A. de; LUMBRERAS, J.F.; COELHO, M.R.; ALMEIDA, J.A. de; CUNHA, T.J.F.; OLIVEIRA, J.B. de. Sistema brasileiro de classificação de solos. 3.ed. rev. e ampl. Brasília: Embrapa, 2013. 353p.

SCHERER, E.E. Doses e formas de aplicação do composto de dejetos suínos no cultivo orgânico de milho e feijão. Revista Agropecuária Catarinense, v.26, p.74-78, 2013.

SCHERER, E.E.; CORTINA, N.; MASSOTTI, Z.; PANDOLFO, C.M. Avaliações agronômica e econômica de uma plataforma de compostagem e do composto produzido. Florianópolis: Epagri, 2009. 49p. (Epagri. Boletim técnico, 150).

SCHERER, E.E.; NESI, C.N.; MASSOTTI, Z. Atributos químicos do solo influenciados por sucessivas aplicações de dejetos suínos em áreas agrícolas de Santa Catarina. Revista Brasileira de Ciência do Solo, v.34, p.1375-1383, 2010. DOI: 10.1590/ S0100-06832010000400034.

SEGANFREDO, M.A.; GIROTTO, A.F. Tratamento dos dejetos suínos e seu impacto econômico em unidades terminadoras. Concórdia: Embrapa Suínos e Aves, 2004. (Embrapa Suínos e Aves. Comunicado técnico, 375).

TEDESCO, M.J.; GIANELLO, C.; BISSANI, C.A.; BOHNEN, H.; VOLKWEISS, S.J. Análise de solo, plantas e outros materiais. 2.ed. Porto Alegre: Universidade Federal do Rio Grande do Sul, 1995. $174 \mathrm{p}$

TIECHER, T.L.; CERETTA, C.A.; COMIN, J.J.; GIROTTO, E.; MIOTTO, A.; MORAES, M.P. de; BENEDET, L.; FERREIRA, P.A.A.; LORENZI, C.R.; COUTO, R. da R.; BRUNETTO, G. Forms and accumulation of copper and zinc in a Sandy Typic Hapludalf soil after long-term application of pig slurry and deep litter. Revista Brasileira de Ciência do Solo, v.37, p.812-824, 2013. DOI: $10.1590 /$ S0100-06832013000300028.

$\overline{\text { Recebido em } 25 \text { de setembro de } 2015 \text { e aprovado em } 24 \text { de fevereiro de } 2016}$

Pesq. agropec. bras., Brasília, v.51, n.3, p.233-242, mar. 2016

DOI: $10.1590 / \mathrm{S} 0100-204 X 2016000300005$ 